\title{
Kein erhöhtes Abortrisiko unter Monotherapie
}

Fragestellung: Führt die Einnahme von Antikonvulsiva während der Schwangerschaft zu vermehrten Aborten oder Totgeburten?

Hintergrund: Epilepsie ist die häufigste neurologische Erkrankung, die eine medikamentöse Dauertherapie auch während der Schwangerschaft notwendig macht. Immerhin betrifft dies 0,3-0,8\% aller Schwangeren. Hinzu kommen Frauen, die aus anderen Gründen regelmäßig Antikonvulsiva (AED) einnehmen, zum Beispiel aus psychiatrischer oder schmerzmodulatorischer Indikation. Bezüglich potenzieller Missbildungsrisiken und Entwicklungsverzögerung der Kinder nach der Geburt liegen inzwischen reichlich Daten vor. Dies ist jedoch nur ein Aspekt in der Beratung von Schwangeren. So stellt sich im Hinblick auf Daten aus Tiermodellen, in denen teilweise deutlich erhöhte Abortraten unter AED zu beobachten waren, die Frage, ob man dieses Problem nicht auch auf den Menschen übertragen muss.

Tomson T, Battino D, Bonizzoni E et al; for the EURAP Study Group. Antiepileptic drugs and intrauterine death. A prospective observational study from EURAP. Neurology 2015; 85: $580-8$
Patienten und Methodik: Die Daten stammen aus dem EURAP-Register, in dem prospektiv Schwangerschaften unter Antiepileptikaexposition europaweit erfasst und bezüglich potenzieller Missbildungen ausgewertet wer- den. Für die vorliegende Studie wurden die sechs am häufigsten verordneten AED in Mono- und Polytherapie ausgewählt und die Schwangerschaften bezüglich Abort oder Totgeburt analysiert.

Ergebnisse: Für die Studie kamen entsprechend den gesetzten Ein- und Ausschlusskriterien 7.055 Schwangerschaften infrage. 1.910 Frauen nahmen Lamotrigin, 1.713 Carbamazepin, 1.171 Valproinsäure, 324 Levetiracetam, 262 Oxcarbazepin und 260 Phenobaribtal in Monotherapie ein. Weitere 1.415 erhielten während der Schwangerschaft eine Polytherapie. 632 der 7.055 Schwangerschaften endeten mit intrauterinem Tod, $592(8,4 \%)$ davon im Sinne eines Spontanaborts, 40 (0,6\%) als Totgeburt. Dabei konnte zwischen den sechs möglichen Monotherapien kein Unterschied festgestellt werden (zwischen 7,9\% und 8,6\%), das Risiko stieg jedoch unter Polytherapie auf 12,1\% relevant an. Ebenfalls erhöht war das Risiko für einen intrauterinen Tod bei älteren Frauen (entsprechend dem bekannten Problem in der Normalbevölkerung), bei bereits stattgehabten Aborten und Totgeburten in früheren Schwangerschaften sowie vorbestehenden kongenitalen Malformationen eines der beiden Elternteile.

Schlussfolgerungen: Epilepsiepatientinnen, die während der Schwangerschaft ein Antikonvulsivum einnehmen, haben kein erhöhtes Abort- oder Totgeburtrisiko. Dies ändert sich unter Polytherapie und, ein wesentlicher Fakt, wenn bereits ein Elternteil eine kongenitale Malformation aufweist.

\section{- Kommentar von Vivien Homberg, Bad Berka}

\section{Weiterhin Entwarnung für Epilepsiepatientinnen}

Im wesentlichen - und das beruhigt - bestätigen die EURAPDaten die im letzten Jahr publizierten Daten des Dänischen Registers und die Rochesterdaten, nun in etwas präziserer Formulierung und Differenzierung in Mono- versus Polytherapie. Damit sind weiterhin vor allem „Begleitfaktoren“ wie Alter der Mütter, kongenitale Vorschädigungen eines der beiden Elternteile oder, wie im Dänischen Register 2014 gut dargelegt, das soziale Umfeld (alleinerziehend, schlechtes Einkommen, geringe Schulbildung, psychiatrische Begleitdiagnosen, Substanzmissbrauch etc.) die wesentlich relevanteren Indikatoren im Bezug auf vorzeitigen, das heißt intrauterinen Tod des Ungeborenen. Möglicherweise sind Epilepsiepatientinnen im Gegensatz zu „normalen“ Frauen ohne besonderen ärztliche Support, auch besserüberpotenzielleRisiken, Verhaltensmaßregeln und den korrekten Umgang mit Antikonvulsiva während der Schwangerschaft aufgeklärt, ordentliche Vorsorgeuntersuchungen eingeschlossen, was Probleme genannter Art reduzieren könnte.

Anhand der aktuellen EUPAR-Daten sollten besonders Polytherapien weiterhin gut überdacht werden, nicht nur was die Totgeburtenrate betrifft, denn auch das Missbildungsrisiko steigt bekanntermaßen bei Einnahme mehrerer AED signifi- kant an. Auf der anderen Seite bleibt bedenkenswert, dass schwere epileptische Anfälle, inklusive eines Status epilepticus nicht gerade förderlich sind für das Ungeborene und sogar je nach Datenlage - Aborte begünstigen können. Wir werden also das Risiko für diese Patientengruppe nicht auf Null herunter setzen können, sollten aber unverändert die üblichen Vorsichtsmaßnahmen und Verhaltensregeln zur Reduktion des Missbildungsrisikos beherzigen: frühe hochdosierte Folsäuregabe, Verteilen der Gesamtdosis auf mehrere Einnahmen, Reduktion der einzelnen Antikonvulsiva auf das absolute Minimum mit Dosisanpassung während der Schwangerschaft.

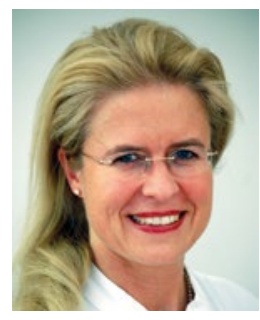

Dr. med. Vivien Homberg, Bad Berka

Chefärztin der Klinik für Neurologie, Zentralklinikum Bad Berka E-Mail:Vivien.Homberg@zentralklinik.de 